\title{
Alternative Methods for the Use of Non- Human Primates in Biomedical Research
}

\author{
Saskia M.Burm ${ }^{1}$, Jan-Bas Prins ${ }^{2}$, Jan Langermans ${ }^{3}$ and Jeffrey J. Bajramovic ${ }^{1}$ \\ ${ }^{1}$ Alternatives Unit, Biomedical Primate Research Centre, Rijswijk, The Netherlands; ${ }^{2}$ Central Animal Facility, Leiden \\ University Medical Centre, Leiden, The Netherlands; ${ }^{3}$ Animal Science Department, Biomedical Primate Research Centre, \\ Rijswijk, The Netherlands
}

\begin{abstract}
Summary
The experimental use of non-human primates (NHP) in Europe is tightly regulated and is only permitted when there are no alternatives available. As a result, NHP are most often used in late, pre-clinical phases of biomedical research. Although the impetus for scientists, politicians and the general public to replace, reduce and refine NHP in biomedical research is strong, the development of 3Rs technology for NHP poses specific challenges. In February 2014 a workshop on "Alternative methods for the use of NHP in biomedical research" was organized within the international exchange program of EUPRIM-Net II, a European infrastructure initiative that links biomedical primate research centers.

The workshop included lectures by key scientists in the field of alternatives as well as by experts from governmental and non-governmental organizations. Furthermore, parallel sessions were organized to stimulate discussion on the challenges of advancing the use of alternative methods for NHP. Subgroups voted on four statements and together composed a list with opportunities and priorities. This report summarizes the presentations that were held, the content of the discussion sessions and concludes with recommendations on 3Rs development for NHP specifically. These include technical, conceptual as well as political topics.
\end{abstract}

\section{Introduction}

Because of their close resemblance to humans, non-human primates (NHP) are employed in biomedical research to study particular aspects of human biology and disease. Like humans, NHP are complex social species and their welfare, housing and care taking is demanding and requires specific expertise and facilities. Both society and biomedical researchers wish to limit the numbers of NHP used for experimental purposes to an absolute minimum, with a maximum effort to ensure animal welfare and good science. Important guidelines for that quest are the three Rs of Replacement, Reduction and Refinement.

In spring 2014 a workshop on "Alternative methods for the use of NHP in biomedical research" was held at the Biomedical Primate Research Center (BPRC) in Rijswijk, The Netherlands. It was organized under the umbrella of the international exchange program of EUPRIM-Net II (http://www.euprim-net. eu) and served four main purposes: i) sharing information in order to accommodate, validate and spread the use of alterna- tive methods in NHP research; ii) discussing the potential and the challenges for advancing the use of alternative methods; iii) identifying obstacles hampering the development of alternatives in NHP research; iv) providing transparency to the general public and other stakeholders on the status of alternative methodology in primate research. The workshop included lectures by key researchers in the field of alternatives discussing progress made on development and implementation of the 3Rs in NHP research. Furthermore, experts from the European Commission and the Dutch government presented the most recent advances on the legislation surrounding NHP research and the 3Rs. Representatives from non-governmental organizations, including animal welfare organizations, legislators, regulators and scientists discussed how the 3Rs in NHP research should be advanced.

The workshop opened with a lecture by Coenraad Hendriksen (Utrecht University and Intravacc) highlighting 3Rs successes in the past as well as stressing the long and winding road ahead for 3Rs development for NHP. After introducing the concept of the 3Rs with practical examples, Hendriksen specified

Received July 21, 2014; Epub July 24, 2014; http://dx.doi.org/10.14573/altex.1406231 
that the development of alternatives for NHP should be considered in the context of the research goals for which NHP are being used. In Europe, $67 \%$ of NHP are used in safety and efficacy regulatory testing, about $15 \%$ in R\&D for medical products and devices and another $15 \%$ in fundamental research. Each of these purposes comes with different opportunities and obstacles for the development of 3Rs methodology, which were discussed. This was illustrated by a success story of NHP replacement for the production of inactivated polio vaccines. In 1960 about 4500 NHP were used annually in the Netherlands for this purpose (Fig. 1). By 1984 this number was brought down to not more than 30 per year as the result of the sequential development of new technology and methodology. Although illustrative for the potential of 3Rs methodology, it took another 23 years to replace these last 30 monkeys, illustrating that the "easier" refinement and reduction targets were fulfilled first and that in particular the last steps required considerable efforts. Hendriksen continued by listing several important dilemmas that pertain in particular to development of 3Rs methodology for NHP. Is replacement by a "lower" animal species really 3Rs methodology? If European restrictions on NHP research become too tight, do we not stimulate outsourcing of NHP research to countries with less historical concern for animal welfare? He stated that the ultimate objective to replace all NHP in biomedical research will not be easy to achieve. As long as society as a whole is riskaversive and not willing to accept the potential negative consequences of denouncing all animal experiments, replacement of NHP research might be the most arduous task to achieve. The focus should therefore be on reduction where possible, and on refinement. Refinement should not be considered as the Cinderella of the 3 Rs and full attention should be given to the diversity of refinement opportunities as they might be easiest to achieve with a relatively high impact on animal welfare.

Friedhelm Vogel (European Federation of Pharmaceutical Industries and Associations (EFPIA); Covance R\&D Laboratories) focused on the role of the 3Rs and NHP in drug development and translational research. He stressed the important role that animal research has had in the process of medicine development, highlighting the role of NHP in particular with a list of examples. There is still an urgent need for new therapies as currently only 10,000 of the known 30,000 diseases can be treated and there is good potential for, e.g., monoclonal antibody-based therapies. Although the use of animals forms a major part of much scientific and medical research, success seen in animal studies has not always translated to the clinic. Many potential drugs fail due to lack of efficacy in humans or concerns about their safety. The likelihood of approval, as assessed after clinical phase 1 development, has declined over the past years (Hay et al., 2014) and needs to be improved by designing more predictive - in vivo - screening methods, including identification and application of appropriate biomarkers. Results from another survey (Olson et al., 2000) support the value of using two species for in vivo toxicology studies to predict human toxicity, as the predictive value is significantly higher in combination. Vogel continued by demonstrating that NHP numbers in toxicity studies can be significantly reduced by different study designs. The "enhanced PPND" study design, as referenced in

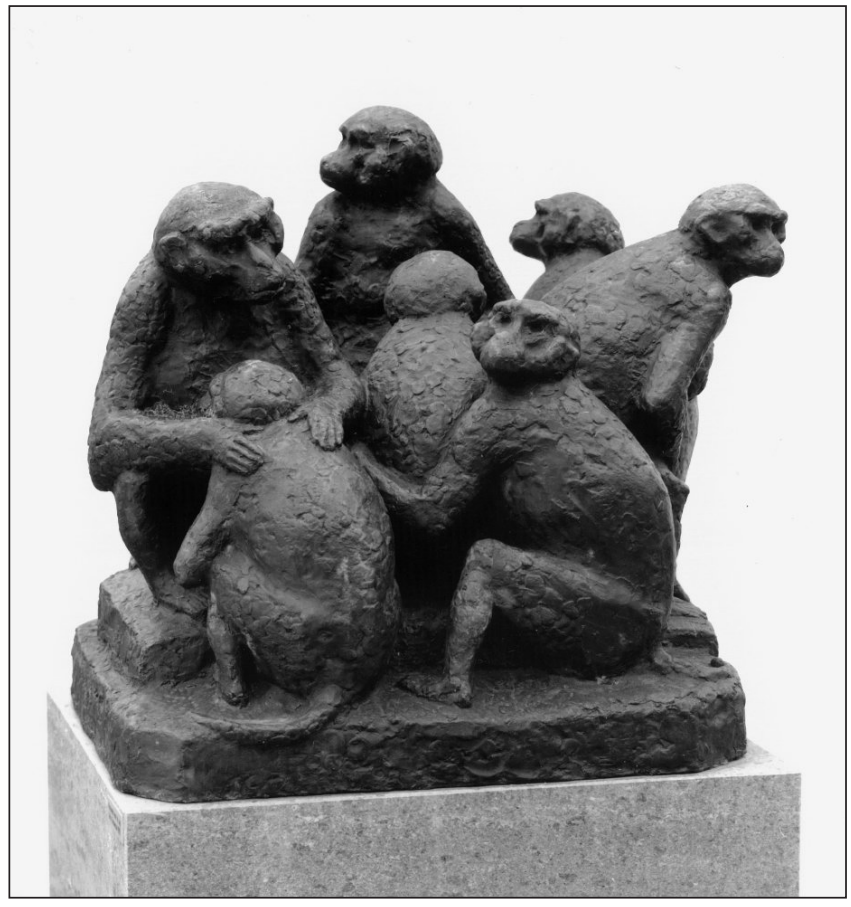

Fig. 1: Monument for the monkey by Arie Teeuwisse (1994) This statue, placed at the campus of the Dutch National Institute of Public Health and the Environment, commemorates the contribution of monkeys to the production of polio vaccines in the past.

the ICH S6 (R1) guideline published in June 2011, was used as an example. He cautioned, however, that group sizes should be kept large enough to have biological significance and statistical power. Thereafter he summarized refinement methodology that has been developed for NHP research including social housing, enrichment, positive reinforcement training, jacketed telemetry, automated activity monitoring and use of only purpose-bred animals. He also offered a glimpse into the future, in which Europe is ageing. He predicted that more research efforts will be dedicated to age-related diseases as well as to personalized medicine, and both will require appropriate and reliable animal models. As the EU is now stimulating the translation of basic research into therapies, this transition will often require the testing of experimental therapies in NHP. On the other hand, the EU wishes to restrict NHP experiments and European researchers are now taking their NHP research outside of Europe, sparking a controversy that is dividing the scientific community. He expressed similar concerns as Hendriksen that the standards of ethical oversight and animal welfare could be lower in those countries compared to those in Europe.

\section{The legal framework and public policy}

Susanna Louhimies (policy officer at the European Commission at DG Environment) discussed EU policy (http://ec.europa. eu/environment/chemicals/lab_animals/home_en.htm) on NHP 
and the 3Rs in the context of Directive 2010/63/EU (EU, 2010). Transposition of the Directive into national legislation of EU Member States should have been completed by November 2012 and the Directive was effective as of January 1, 2013. Not all Member States managed to meet these requirements in time, including Italy, the Netherlands, Poland and Romania (see also below). Although existing stricter measures could be maintained, new, more stringent measures can no longer be adopted by Member States.

EU legislation explicitly states that the use of NHP is still necessary in biomedical research, but raises ethical concern and practical problems in terms of meeting their behavioral, environmental and social needs in a laboratory environment. The EU acknowledges that the use of NHP is of great concern to the public. The Directive spells out the 3Rs and makes their application a legal requirement at all times. In addition, the application of refinement is not limited to scientific procedures but is also relevant in relation to care, accommodation and breeding of animals. Finally, the Directive requires that more resources should be made available for the development, validation and promotion of the 3 Rs.

The Directive restricts the use of NHP in relation to species used, areas of use and who benefits from the research. The use of great apes is prohibited in the EU. Under exceptional circumstances a temporary exemption can be granted for the purposes of research aimed at the preservation of those species and where action is needed in case of an unexpected outbreak of a life threatening, debilitating condition endangering human beings. Furthermore, such a temporary measure will be put to vote by a Member State committee to either authorize or revoke it. The use of other species of NHP is restricted to basic research, preservation of the species and applied research for human health and diseases.

The 3Rs and NHP are specifically addressed with regard to the source, use, care and accommodation of NHP, follow-up, reporting and controls. As capturing of NHP from the wild is stressful and elevates the risk of injury and suffering, it is the ambition of the EU to use only second-generation (F2) purpose-bred NHP, ultimately from self-sustaining colonies. A feasibility study on the use of F2 animals will be initiated and results should be published by November 2017. Furthermore, results from a feasibility study on sourcing from self-sustained colonies should become available by November 2022. NHP should be socially housed, taking into account the need for environmental complexity and enrichment (e.g., foraging), and training of the animals. Each NHP must have an individual history file from birth onward to be able to assess and provide the care, accommodation and treatment adjusted to its individual needs and characteristics.

A severity assessment framework is put in place to ensure that the $3 \mathrm{Rs}$ are considered and implemented during all phases of a project. All projects using animals will undergo a systematic project evaluation. To improve transparency and to follow up on NHP research and implementation of the $3 \mathrm{Rs}$, the EU requires non-technical project summaries and systematic retrospective assessments of all projects using NHP. Animal welfare bodies and national committees will play an important role in the dissemination of information on the 3Rs. Member States are required to publish annual statistics. The statistical reports will also include information on sourcing of NHP. Finally, the national authorities perform annual inspections of establishments breeding, supplying or using NHP. The frequency of inspections should be adapted on the basis of risk assessment.

The EC will review the Directive by 2017 , taking into account the progress made towards replacing the use of animals and, in particular, NHP. Where appropriate, thematic reviews will be conducted on the replacement, reduction and refinement of animal use, paying specific attention to NHP, technological developments, and advances in science and animal-welfare knowledge. The scientific community will be fully involved in these reviews. Louhimies concluded by stating the importance of all forms of communication, from scientist to scientist, but also with the public.

Angelique Nielen (policy officer at the Dutch Ministry of Economic Affairs) presented how the Netherlands is implementing the new EU Directive. Although the EU Directive 2010/63/EU implied changes in national law and secondary legislation, it allowed maintenance of existing stricter measures as was the case in the Netherlands. The Dutch Act on animal experimentation was and is based on a "Not allowed, unless there is no alternative" principle, whereas the EU Directive is based on a "Permitted, provided that special requirements are met" principle. In addition, in the Netherlands a total ban on the use of great apes for animal testing exists since 2003. It was decided to keep this ban in place. She next presented data demonstrating that only a small proportion of experiments in the Netherlands involve new world $(<0.1 \%)$ or old world monkeys $(0.1 \%)$. Those were mainly obtained from registered breeding or supplying establishments within the Netherlands, and some were re-used from other experiments. The national government aspires to replace the use of animals in experimental procedures by other methods, but acknowledges that the use of animals is still necessary to protect human and animal health and the environment. The ambition of the Dutch government is to obtain joint responsibility of public and private parties, to increase transparency on animal testing and to establish international collaborations. Key issues for the Dutch government are the central authorization of procedures, the issue of purpose-bred animals and how to minimize pain, suffering and distress of the animals used.

Marianne Kuil (senior policy advisor on animal experiments and biotechnology at the Dutch Society for the Protection of Animals (DSPA)) started her presentation by presenting animal experimentation in the context of a chain of stakeholders rather than approaching it as a single event. That chain consists of biomedical research organizations, industry, government and authorities, patients and patient organizations, fundraising organizations, medical and veterinary students, doctors and healthcare workers, the general public and animal protection/welfare organizations. All these stakeholder groups should be consulted, have an informed opinion, be transparent and share responsibility for animal experiments, including the general public and fundraising organizations. She emphasized 
that the use of animals for scientific purposes, and especially the use of NHP, is of major concern to the general public. She made clear that different animal welfare organizations differ in their viewpoints. The DSPA recognizes the intrinsic value of all animals (it does not distinguish between species) and states that all use of animals for scientific purposes should be forbidden. However, DSPA is realistic in recognizing dilemmas that are raised by this viewpoint and is seeking the way forward through dialogue based on respect, trust, involvement and hope and by the realization that partners in the chain have common goals. Furthermore, Kuil emphasized the importance of communication to the public at large about the use of NHP and the development of the $3 \mathrm{Rs}$ and encouraged the audience to think about how communication with the public can be improved.

\section{Advances in 3Rs methods for housing, colony management and training}

Jan Langermans (BPRC, The Netherlands) and Karolina Westlund (Karolinska Institute, Sweden) discussed advances in refinement of housing, colony management and training of NHP. To refine NHP housing and breeding the specific biological needs of each species should be taken into consideration, including their social system. Natural grouping patterns of macaques include large multi-male and multi-female groups with dominance hierarchy, from which the males migrate to other groups when they become sexually mature. Natural grouping patterns of marmosets include monogamous pairs with their offspring. To obtain stable, self-sustaining breeding groups, natural grouping patterns and behavior must be accommodated as much as possible to enhance welfare and to reduce stress, thereby possibly also leading to better science. However, for scientists this provides challenges, including difficulties when introducing new males into established rhesus macaque groups, rank-associated fighting between grouphoused animals and exposure to the environment, including potential pathogens. Social housing of NHP should also consider natural environment and behaviors, including floor bedding that enables foraging, availability of an outside compartment, sufficient height of the facilities to allow climbing and jumping, and mimic a tropical climate for marmosets. Social housing of NHP in laboratory settings has positive effects on the behavior and well-being of the animals (DiVincenti and Wyatt, 2011). Langermans presented data that periods of stress and stereotypic behavior are significantly lower in animals that were housed socially and in the presence of enrichment (bedding), strongly suggesting that these factors also contribute to "better science" (Louwerse et al., in preparation). He also advocated the use of the enrichment manual (Vernes and Louwerse, 2010) written and edited during EUPRIM-Net (info via http://www.bprc.nl) and introduced the concept of positive reinforcement training (PRT). The basic principle of PRT is rewarding and reinforcing specific trained behavior to encourage the animal to perform a specific task in response to a trained stimulus.
Karolina Westlund was involved in the development of the EUPRIM-Net Seminar Group Lectures that were developed to give seminars on behavior management techniques. As she demonstrated, clicker training - an example of PRT- strongly reduces the stress level of animals used during experimental procedures. Training is thus not only important from an ethical perspective but also improves the quality of science. Specific PRT features were demonstrated by showing the DVD that was recorded under the umbrella of EUPRIM-Net: "Training laboratory animals" (info via http://www.euprim-net.eu). Training includes different phases from habituating naive animals to the trainer and teaching some basic operant behaviors to full compliance in difficult procedures. Challenges of positive reinforcement training are amongst others the requirement of a serious time investment of the trainers and that not all animals are good trainees. Westlund showed that progress has been made in delineating which personality dimensions are correlated to trainability of the animals and what other factors influence training time investment.

\section{Advances in 3Rs methods for neuroscience and neuroimmunology}

Michael Niessing (German Primate Centre, Germany) presented data on the development of a novel model that allows setting up cognitive tasks and recordings of behavioral data in NHP without movement restrictions. This is a major improvement of the conventional setup of experiments in cognitive neuroscience where animals are usually restricted in several ways. The model comprises a cage-based system (eXperimental Behavioral Instrument, XBI) and utilizes a computer controlled touch screen and rewarding system that NHP can interact with in an unrestrained and self-paced manner. It can be combined with miniaturized wireless devices for neuronal recordings to perform cognitive neuroscientific experiments. Data and video clips demonstrated that this system stimulates the natural curiosity of NHP. In that respect, the system itself may be regarded as part of environmental enrichment. Challenges remain, such as the addition of an automatic identification system which is of particular importance if the freely moving monkeys are to be group-housed. Finally, Niessing showed data on efforts to predict which animals are most likely to enjoy participation and learning, as not all animals have similar potential.

Saskia Burm (BPRC, The Netherlands) presented novel in vitro NHP models that have been established to study neurodegenerative diseases. These can be initiated from surplus brain material (Fig. 2) that becomes available from animals that have been sacrificed for other reasons (Zuiderwijk-Sick et al., 2007). These in vitro NHP models include organotypic brain slice cultures, mixed glial cell cultures, dissociated primary cell cultures and co-cultures of glial cells with cells of the adaptive immune system. Data were presented on how these in vitro models can aid in the refinement, reduction and replacement of in vivo models for neurodegenerative diseases. They can be used as a pre-screening tool of drugs and/or they can complement in vivo models by dissecting complex biological 


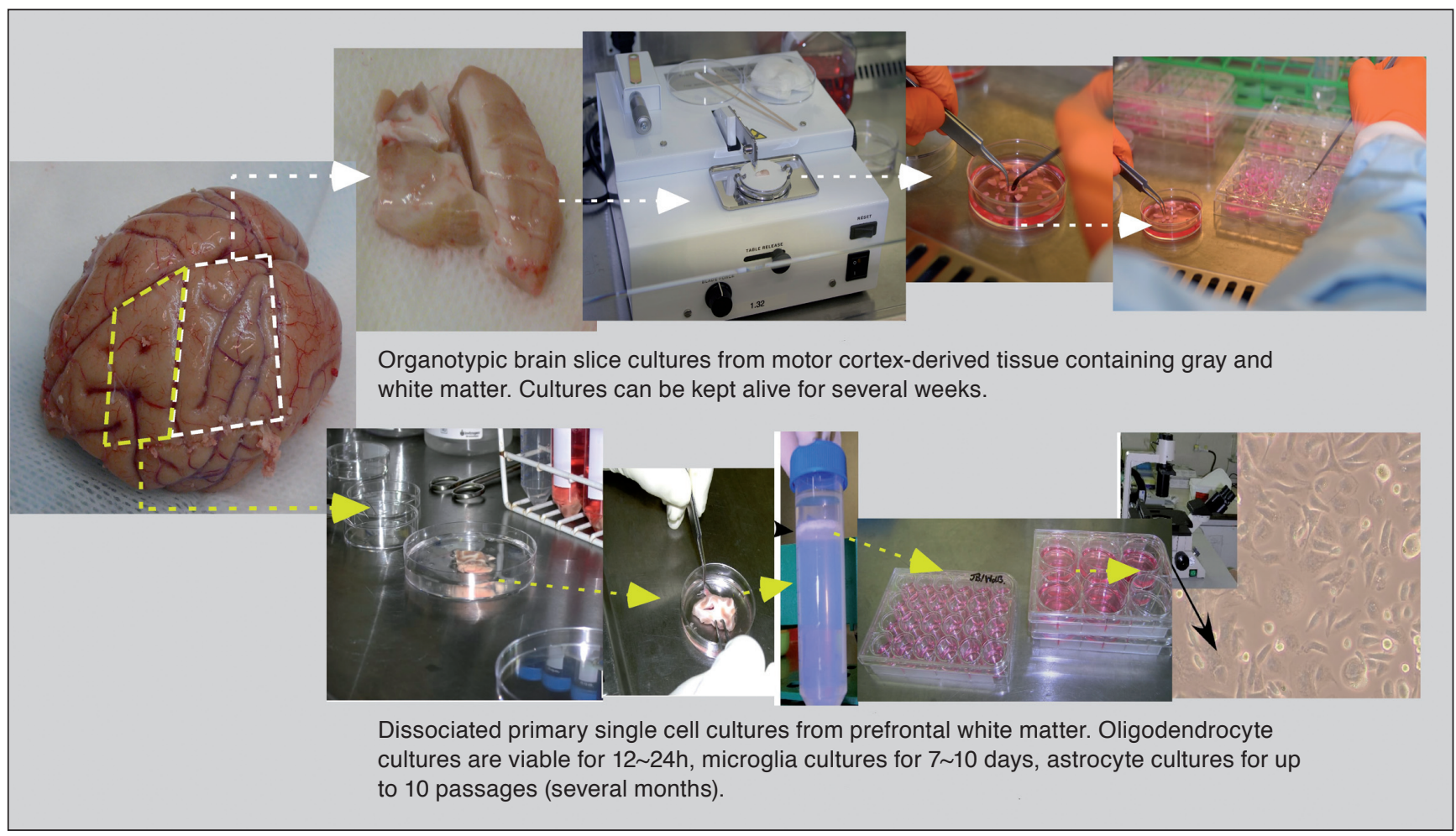

Fig. 2: Alternatives for in vivo central nervous system research

Rhesus macaque brain tissue is acquired with minimal post mortem times and processed for different in vitro purposes. Motor cortex is used to initiate organotypic brain slice cultures, prefrontal subcortical white matter is used to initiate dissociated brain cell cultures. Brain tissue is always derived from animals that were sacrificed for other purposes than initiating in vitro cultures.

mechanisms that are difficult to study in vivo, which may lead to the discovery of new therapeutic targets (van der Putten et al., 2009, 2012). She also discussed the advantages and disadvantages of these NHP primary in vitro models compared to those that exist for humans and rodents (Smith and Dragunow, 2014).

\section{Advances in 3Rs methods for toxicology}

Bas Blaauboer (Institute for Risk Assessment Sciences, The Netherlands) explained the paradigm shift that is now taking place in risk assessment that will hopefully lead to a reduction of NHP use in safety studies. The classical way to assess safety was by exposing animals to compounds and to measure acute toxicity, repeated dose toxicity and a number of more specialized toxic endpoints, such as reproductive and genotoxic effects. An important quantification parameter is the No Observed Effect Level (NOEL) as the most sensitive endpoint, which then needs to be extrapolated to establish safety standards for human exposure. Extrapolation takes into account safety factors for uncertainty. However, as long as the mechanism of action of the compounds is unknown, extrapolation remains estimated guesswork. Toxicity is characterized by the critical concentration and time of exposure (dose metric) to the critical compound at the site of action. The new paradigm in toxicology takes these fac- tors into account. An important catalyzer of this paradigm shift is the 2007 report of the US National Research Council (NRC, 2007). If exposure levels to compounds are predictably low, exposure-based waiving of risk assessment can be considered. For certain categories of chemicals this is now done making use of the Threshold of Toxicological Concern (TTC) concept.

Furthermore, the potential of compounds to activate stress response pathways, toxicity pathways and adverse outcome pathways can be evaluated in vitro by assessments that are mechanism-based. In silico methods (QSARs, systems biology, pathway modeling, PBPK and PKPD modeling) and in vitro methods (testing battery for both toxicological endpoints and kinetic parameters) can be used in an integrated scheme. Integration of all available data in a stepwise (hierarchical) approach improves the transparency and efficacy of the risk assessment process and will reduce the number of animals needed in safety testing. Challenges remain, such as the selection of the most important parameters to assess risk and the assessment of what can be called no effect, adaptive effects or adverse effects of a compound (Blaauboer et al., 2012).

Kathryn Chapman (NC3Rs, UK) introduced the National Centre for 3Rs (NC3Rs), an independent scientific organization that was established by the UK government to support the science base through the application of the 3Rs. They do so by funding relevant research, by creating new industry/academic partnerships, by creating forums for pre-competitive data shar- 
ing and by influencing regulators and politicians. Over the last years, NC3Rs have organized workshops, brought together working groups and published on their findings. Regarding NHP, NC3Rs have focused on the role of NHP in the development of monoclonal antibody (mAb)-based therapies, and on study design of chronic toxicity and reproductive toxicity studies that use NHP (Chapman et al., 2009, 2010, 2012, 2013). For mAb-studies, it should be noted that NHP are not necessarily the primary species of choice. There are circumstances where pre-clinical studies can be performed in rodents. The revision of the European guideline for preclinical safety evaluation of biotechnology-derived pharmaceuticals (ICH S6: http://www. ich.org/fileadmin/Public_Web_Site/ICH_Products/Guidelines/ Safety/S6_R1/Step4/S6_R1_Guideline.pdf) leaves open the possibility to use one species if its toxicity profile is similar to that of humans. Chapman next summarized recommendations for study design of toxicity studies using NHP. Often the number of dose groups as well as the number of recovery animals can be reduced without affecting the outcome. She calculated that, for a total toxicity program, NHP numbers can be reduced from 144 to 52 by redesigning the study following NC3Rs guidelines. NC3Rs will continue funding and collaborating to advance the development and application of nonanimal methods. She also commented on a recently published letter that questioned the added value of NHP in the development of mAb therapies (van Meer et al., 2013). The letter was based on a study for which data were used of EMA-registered drugs only, rather than those drugs which had been terminated from development prior to regulatory submission. This may have biased the outcome. NC3Rs is currently collecting data from unpublished compounds that may have been dropped because of adverse effects observed in NHP. She concluded by stating that there are further opportunities to reduce NHP use, but that these have to be science-driven to ensure human safety. NC3Rs will continue their role in cross-company collaborations as these give bigger - anonymized - datasets and a stronger voice. NC3Rs provides an honest-broker role between companies and regulators and wishes to advocate the $3 \mathrm{Rs}$ as a catalyst for change.

\section{Advances in 3Rs methods for vaccines/immunology}

William Warren (Sanofi Pasteur, US) introduced the MIMIC ${ }^{\circledR}$ system. This in vitro model mimics the human immune system and can be used to study immunophysiology of neonates, adults, the elderly and those with allergies, but also to assess immunogenicity and functionality of drugs and new vaccines. Human immune cells are placed into engineered tissue constructs that seek to emulate the physiological environment of the human immune system in modular microtiter wells. The MIMIC ${ }^{\circledR}$ system is modular and consists of a peripheral tissue equivalent (to mimic local innate immune responses), a lymph node tissue equivalent (to mimic and study adaptive immune responses) and functional assays (to, e.g., assess neutralization). All mod- ules have been developed and characterized in terms of cell types, cell numbers, kinetics, etc. and provide the possibility to study immune responses in vitro in an outbred system. The MIMIC ${ }^{\circledR}$ system can be used as a high throughput screening tool to study functionality of various compounds, thereby possibly reducing the number of animals needed to assess drugs, vaccines and biosimilars. Warren demonstrated that data from the MIMIC ${ }^{\circledR}$ system correlate well with clinical data for several vaccines and biologicals. Ultimately the system would be akin to running "clinical trials in test tubes" to better downselect candidates before entering into the clinic. At this moment there are no plans to develop similar ex vivo technology for NHP.

Ingo Spreitzer (Paul Ehrlich Institute, Germany) discussed the monocyte activation test (MAT) that has been developed to replace classical in vivo pyrogenicity tests in rabbits. Pyrogenicity testing is required for many biologicals, and is also an adverse effect frequently encountered during adjuvant development. The MAT is based on the principle that well-characterized pyrogens (such as WHO reference lipopolysaccharide (LPS)) induce the secretion of interleukin (IL) IL-1 $\beta$, IL- 6 and tumor necrosis factor (TNF) $\alpha$ from human as well as from NHP-derived monocytes. Spreitzer presented data showing that pooling individual blood samples (and cryopreservation if desired) is a good method to standardize and to minimize variability due to the outbred nature of humans and NHP. Cryopreservation procedures of blood from different NHP species require different methodology and it remains to be established which NHP mimics human fever responses the best. Besides replacement and reduction, species-specific MATs might help to refine foreseen in vivo experiments (e.g., evaluation of new antigen/adjuvant compositions). NHP MATs, in combination with in vivo experiments, can serve to carefully correlate in vitro and in vivo data, thereby contributing to the acceptance and application of the MAT.

Ulrike Sauermann (German Primate Centre, Germany) presented on the development of an in vitro NHP model to assess AIDS vaccine efficacy. This is currently done in vivo by repeated exposure of vaccinated NHP to low doses of SIV/SHIV with the aim to finally infect all animals. A simple, reproducible and standardized in vitro test would not only ultimately lead to a reduction of NHP used, but also refine animal experiments, since conventional assessments of immune parameters are not informative about AIDS vaccine efficacy. Sauermann presented data showing that the ratio of MX1/CXCL10 RNA levels in lymphocytes as well as plasma IP-10 levels correlated with numbers of exposures required for infection. The markers for interferon (IFN)- $\gamma$ - and IFN- $\alpha$-mediated immune stimulation also correlated with SIV-specific CD4 T cell responses. These putative correlates of protection can be used to determine AIDS vaccine efficacy ex vivo within $48 \mathrm{~h}$ post vaccination. However, the protocol for in vitro SIV-infection of lymphocytes to predict AIDS vaccine efficacy requires further optimization, with a special focus on the in vitro activation procedure of lymphocytes as this appears to be critical.

Jeffrey Bajramovic (BPRC, The Netherlands) presented integrated in vitro test systems that were developed to aid adju- 
vant development. Adjuvants are formulations which upon administration lead to non-specific immune stimulation. They are used to induce immune responses directed against pathogens (as for vaccination purposes) or to generate immune responses against components of the body itself (as in experimental animal models of human auto-immune diseases, e.g., multiple sclerosis, diabetes or rheumatoid arthritis). However, some of the more potent adjuvants are notorious for their adverse effects. Most notable is the development of granulomatous skin lesions, causing varying degrees of discomfort to animals in biomedical experiments. There is therefore an urgent need for new improved adjuvants. The integrated in vitro testing strategy for the development of new adjuvants is comprised of luminescent bioassays for Toll-like receptor-mediated responses to assess adjuvanticity (Bsibsi et al., 2010; Boele et al., 2009; Jagessar et al., 2010; Koopman et al., 2013) and 2D/3D in vitro granuloma models to assess adverse effects. Data were shown demonstrating that the sequential use of multiple bioassays as a robust integrated test system can rapidly generate detailed information and can aid the development of new potent adjuvants with minimal adverse effects. Preliminary data on new adjuvant candidates for NHP with less adverse effects showed that in vitro results correlated well with in vivo findings. Although this project contributes mainly to the $\mathrm{R}$ of Refinement, adjuvants with serious adverse effects are used for biomedical research in many different animal species. The development of improved adjuvants might therefore also positively affect the welfare of those species.

\section{Advances in 3Rs methods: stem cells and biobanking}

Robert Passier (Leiden University Medical Centre, The Netherlands) described the use of stem cells as a 3Rs alternative for animal experiments. Stem cells can be derived from human and NHP origin. They are classically divided into adult stem cells (found in many organs), embryonic stem cells and induced pluripotent stem cells (iPSC). Adult human stem cells have limited differentiation and proliferative capacity. Embryonic stem cells are derived from blastocyst stage embryos, are pluripotent and have high proliferative capacity. However, obtaining these stem cells remains an ethically sensitive topic. iPSC are obtained by reprogramming somatic cells (by the addition of genetic material to, e.g., skin fibroblasts, or by culturing them in special culture media). Because of their origin they are free from ethical concerns. iPSC can form all tissues of the body and can be expanded and maintained in culture. These cells can be used in regenerative medicine, but also in toxicological studies, disease models and drug screening. Passier gave an overview of differentiation regimes (leading to, e.g., beating cardiomyocytes) and the possibilities of using different types of stem cells (e.g., for transplantation purposes). He argued that iPSC protocols should also be further developed for NHP as evaluation is necessary for critical assessment of iPSC clinical feasibility and safety, specifically mentioning the potential to test and validate autologous stem cell-based therapies. NHP-derived iPSC also provide researchers with 3 Rs opportunities of great potential for in vivo NHP models.

Nadège Devaux (Rousset Station de Primatology, France) presented on the development and management of a new NHP biobank. The purpose is to collect, characterize and distribute high-quality tissues, cells and biological samples (DNA, RNA, proteins) of accredited primate centers and laboratories with accompanying behavioral and veterinary information. They have collected samples of many organs and of specific CNS areas from macaques, African green monkeys and marmosets. Further developments included preparation and storage of sperm, oocytes and cells from reproductive organs for several applications (genetic studies, in vitro fertilization and cryopreservation of gametes from endangered NHP species). Devaux explained the quality control checks that are important for proper biobanking as well as the necessity of good registration systems. She also summarized the public and private laboratories that have made use of the biobank thus far and stressed the importance of networks like EUPRIM-Net in supporting biobanks.

\section{Opportunities and challenges}

An important goal of the workshop was to discuss the opportunities and priorities for 3Rs development in biomedical research with NHP. To facilitate discussion, subgroups were formed. Every group was presented with four statements and each member of the group was asked to rank the four statements. In addition, subgroups were asked to compose a list of opportunities and priorities. Results were discussed in a plenary session and are summarized below.

Recent advances in technology (CRISPR/CAS9) now render it possible to generate transgenic animals with much higher efficacy. This opens the route towards generating and using transgenic NHP, and recently a first paper was published on transgenic NHP (Niu et al., 2014) demonstrating the potentialIs this a $3 R$ s opportunity?

None of the groups considered the generation of transgenic NHP a 3Rs opportunity. Firstly, concerns were raised whether the public at large would accept the development of transgenic NHP models. Secondly, based on experience with transgenic mouse studies, it was estimated that the availability of transgenic NHP would not contribute directly to replacement, reduction or refinement. Thirdly, because of the outbred nature of NHP, results from studies that use transgenic NHP should always be interpreted very carefully as genotypic differences will also contribute importantly to study outcome. Although groups agreed that in the long run better control and a reductionist approach to correlations between gene function and pathology could lead to better science, this indirect 3Rs effect was considered of minor importance. In the general discussion it was suggested that it might be interesting to have the practicality and the ethics of this technology assessed in detail to generate a more informed opinion regarding the subject. 
Stem cell technology - Do we need NHP stem cells and is this a 3 Rs opportunity?

All groups considered stem cells an opportunity to reduce and replace the number of NHP. Not only can these cells supplement in vivo studies with in vitro opportunities in the form of integrated testing schemes, but they can also be used in backwards validation studies with in vivo models. This would teach us about the validity of human stem cells as predictive models. Especially, induced pluripotent stem cells provide 3Rs opportunities, since these can be generated from, e.g., fibroblasts that can be obtained with minimal discomfort. Furthermore it was put forward that there are still many basic scientific questions regarding stem cells for which the comparison of NHP with human stem cells would be informative. Finally, it was discussed that the development of personalized - regenerative - medicine will probably require preclinical testing in NHP, adding an additional argument in favor of further development of NHP stem cell technology (Hong et al., 2014; Kamao et al., 2014; Morizane et al., 2013).

Biobanking - Do we need biobanking for NHP and is this a $3 R$ s opportunity?

All groups considered biobanking a true 3Rs opportunity. Biobanks could directly lead to a reduction in NHP numbers necessary for in vivo experiments, but also lead to better science by providing researchers with additional opportunities to test, e.g., biologicals, on tissue or in vitro first. Some concerns were raised regarding how biobanking should be financed as most funding is project-related and biobanking is an infrastructural issue. Secondly, the question was raised if there was a finite number of individuals per species of which biological materials should be banked and whether active biobanking could be turned into passive biobanking when frozen and stored collections are of sufficient size. The general opinion was that we have not reached that point yet, but the point is - as it is for humans - valid. Collaboration of various existing biobanks and communication on the availability of NHP materials also provides further opportunities on implementation of the 3 Rs.

\section{Should all 3Rs be considered equal for NHP?}

Groups were unanimously positive. Equal attention, focus and budget should be allocated to work on the development and implementation of all 3Rs alternatives including refinement initiatives, which are often aimed at realistic goals. Different opinions were expressed on whether the replacement of NHP by other species was desirable and whether this is a 3Rs opportunity. The DSPA stated that they consider and value every animal, species-independent, as equal. When replacement and reduction options are exhausted or out of reach, refinement options should be developed as much as possible. In particular for NHP, refinement might often be the most achievable $\mathrm{R}$ and should not be regarded as less than the other two Rs.

3Rs opportunities listed by the different groups could be divided into different categories. First, groups focused on recent technological advances and the possibility to translate such technologies to NHP. 3D printing and 3D cell culture, NHP biomimetic systems (such as the abovementioned MIMIC ${ }^{\circledR}$ system) and stem cells were discussed in some depth and were high on the lists. However, as for the possibilities of transgenic NHP, thorough technological assessments are needed to assess practicality. Second, most groups recognized 3Rs potential in the further development and validation of NHP in vivo models. Especially, additional studies of compounds/vaccines with discrepant results between pre-clinical studies in NHP models and clinical assessment in humans would be desirable. If certain NHP models turn out to have limited predictability, these models should be optimized, changed or abandoned. Although there was consensus on the importance of this topic, there was also consensus on the difficulties to get such - expensive - efforts funded. Maybe there could be a role for pre-competitive platforms financed by pharmaceutical companies here. Along the same line, the compilation of a registry of failed compounds was discussed. Such compounds are not tested in humans, and the development pipeline stops after pre-clinical assessment in NHP. Not only is this information valuable for other NHP researchers and could it reduce NHP numbers by preventing unnecessary studies, such a registry could also aid the development and validation of better NHP models. Research on why compounds fail should be stimulated in order to gain understanding and to learn from these failures. A data collection and registration system was proposed and NC3Rs mentioned that they are already working on this topic. NC3Rs and other groups saw additional 3Rs possibilities in the improvement of NHP safety study design (Chapman et al., 2012, 2013). Although much of this work has been published, not all companies have adapted their study designs accordingly. Extra and continuous efforts are necessary to push this topic further. Finally, most groups recognized 3Rs possibilities in communication, education and outreach. For refinement of NHP research, seminar group lectures have been developed on topics regarding animal behavior management, ranging from breeding laboratory primates to enrichment and problem solving. The development of specific e-learning platforms and expert technical workshops may provide further opportunities for disseminating $3 \mathrm{Rs}$ technology. It was stressed that communication should not be restricted to scientists but that there should be active efforts to get the general public, regulators and fund-raising charities informed and involved. As long as animal experiments are still necessary, the responsibility should be shared and not be left to the researchers alone. Communication, outreach and dissemination of the 3Rs should aim for Europe and beyond. A dilemma that was brought up several times is that the progress that is made in implementing the 3Rs for NHP research in industrialized countries leads to higher costs as compared to emerging countries with lesser standards on animal welfare. However, it should be stressed that physically and mentally fitter animals make better science. Despite this, researchers and companies can escape EU legislation on NHP research and benefit economically by outsourcing NHP experiments to emerging countries, which is indeed happening more and more often. This tendency might ultimately lead to decreased implementation 
of the 3Rs in NHP research worldwide, and measures that are taken in Europe in that respect might be counterproductive. Efforts must therefore also be made to effectively communicate the implementation of the 3Rs in NHP research from industrial countries to emerging countries.

Evaluation of the workshop learned that attendees appreciated the mix of presentations on legal, scientific and public opinion topics and that there is a necessity for joint efforts to secure resources to advance 3 Rs development. Although the public at large and governments agree on the desirability of $3 \mathrm{Rs}$ research, dedicated budget is limited. Workshops like this might not only advance sharing of knowledge but the informed discussions might also aid decision makers in their process of prioritizing limited research budgets.

\section{Recommendations}

The use of NHP in biomedical research is much debated and there is a strong impetus to develop 3Rs technology for NHP. Initiatives such as EUPRIM-Net remain essential for continuing education and improvements in the development of the 3Rs in NHP research. This report reflects the opportunities and challenges as experienced by those involved. Although by no means meant to discourage other initiatives, we would recommend that the following topics should receive full attention:

- Refinement: Refinement initiatives for NHP often aim for realistic goals. Equal attention, focus and budget should be allocated to work on the development and implementation of all 3Rs alternatives.

- Induced pluripotent stem cell technology for NHP: Induced pluripotent stem cells can provide researchers with valuable 3Rs opportunities. They can be generated from cells that can be obtained with minimal discomfort to the animal and without ethical concerns. They can be used to generate all cell types of the body for in vitro studies. Such studies can supplement NHP in vivo studies, and they can also teach us about the validity of human stem cells as predictive models.

- Further development, optimization and validation of NHP in vivo models: NHP in vivo models should be critically evaluated for their predictive value. Performing in vivo studies of compounds/vaccines with discrepant results between preclinical studies in NHP models and clinical assessment in humans should be stimulated in backwards validation studies as a means to improve NHP models.

- Registry of failed compounds: Compounds that fail during pre-clinical testing are not tested in humans. This information is valuable for other NHP researchers and could reduce NHP numbers by preventing unnecessary studies.

- Communication, education and outreach: Communication about the 3Rs and NHP research should not be restricted to scientists. Instead, there should be active efforts to get the public at large, regulators and fund-raising charities informed and involved. Responsibility for performing and communicating about NHP research should be shared. Communication, outreach and dissemination of the 3Rs should be global as increasing restrictions on NHP research, e.g., in Europe, might be counterproductive by stimulating outsourcing of NHP research to countries with less concern for animal welfare.

\section{References}

Blaauboer, B. J., Boekelheide, K., Clewell, H. J. et al. (2012). The use of biomarkers of toxicity for integrating in vitro hazard estimates into risk assessment for humans. ALTEX 29, 411-425. http://dx.doi.org/10.14573/altex.2012.4.411

Boele, L. C., Bajramovic, J. J., de Vries, A. M. et al. (2009). Activation of Toll-like receptors and dendritic cells by a broad range of bacterial molecules. Cell Immunol 255, 17-25.

Bsibsi, M., Bajramovic, J. J., Vogt, M. H. et al. (2010). The microtubule regulator stathmin is an endogenous protein agonist for TLR3. J Immunol 184, 6929-6937. http://dx.doi. org/10.4049/jimmunol.0902419

Chapman, K., Pullen, N., Coney, L. et al. (2009). Preclinical development of monoclonal antibodies: considerations for the use of non-human primates. MAbs 1, 505-516. http://dx.doi. org/9676 [pii]

Chapman, K. L., Pullen, N., Andrews, L. et al. (2010). The future of non-human primate use in mAb development. Drug Discov Today 15, 235-242. http://dx.doi.org/10.1016/j. drudis.2010.01.002

Chapman, K. L., Andrews, L., Bajramovic, J. J. et al. (2012). The design of chronic toxicology studies of monoclonal antibodies: implications for the reduction in use of non-human primates. Regul Toxicol Pharmacol 62, 347-354. http://dx.doi. org/10.1016/j.yrtph.2011.10.016

Chapman, K. L., Holzgrefe, H., Black, L. E. et al. (2013). Pharmaceutical toxicology: designing studies to reduce animal use, while maximizing human translation. Regul Toxicol Pharmacol 66, 88-103. http://dx.doi.org/10.1016/j.yrtph.2013.03.001

DiVincenti, L., Jr. and Wyatt, J. D. (2011). Pair housing of macaques in research facilities: a science-based review of benefits and risks. J Am Assoc Lab Anim Sci 50, 856-863.

EU - European Union (2010). Directive 2010/63/EU on the Protection of Animals Used for Scientific Purposes. Off J Eur Union L 276, 33-79. http://eur-lex.europa.eu/legal-content/ EN/TXT/PDF/?uri=CELEX:32010L0063\&rid=1

Hay, M., Thomas, D. W., Craighead, J. L. et al. (2014). Clinical development success rates for investigational drugs. Nat Biotechnol 32, 40-51. http://dx.doi.org/10.1038/nbt.2786

Hong, So G., Winkler, T., Wu, C. et al. (2014). Path to the clinic: Assessment of iPSC-based cell therapies in vivo in a nonhuman primate model. Cell Reports 7, 1298-1309. http://dx.doi. org/http://dx.doi.org/10.1016/j.celrep.2014.04.019

Jagessar, S. A., Kap, Y. S., Heijmans, N. et al. (2010). Induction of progressive demyelinating autoimmune encephalomyelitis in common marmoset monkeys using MOG34-56 peptide in incomplete freund adjuvant. J Neuropathol Exp Neurol 69 , 372-385. http://dx.doi.org/10.1097/NEN.0b013e3181d5d053

Kamao, H., Mandai, M., Okamoto, S. et al. (2014). Characterization of human induced pluripotent stem cell-derived retinal 
pigment epithelium cell sheets aiming for clinical application. Stem Cell Reports 2, 205-218. http://dx.doi.org/10.1016/ j.stemcr.2013.12.007

Koopman, G., Beenhakker, N., Burm, S. et al. (2013). Whole blood stimulation with Toll-like receptor (TLR)-7/8 and TLR9 agonists induces interleukin-12p40 expression in plasmacytoid dendritic cells in rhesus macaques but not in humans. Clin Exp Immunol 174, 161-171. http://dx.doi.org/10.1111/ cei. 12155

Morizane, A., Doi, D., Kikuchi, T. et al. (2013). Direct comparison of autologous and allogeneic transplantation of iPSC-derived neural cells in the brain of a nonhuman primate. Stem Cell Reports 1, 283-292. http://dx.doi.org/10.1016/ j.stemcr.2013.08.007

NRC - National Research Council (2007). Toxicity Testing in the $21^{\text {st }}$ Century: A Vision and a Strategy. Washington, DC, USA: The National Academies Press.

Niu, Y., Shen, B., Cui, Y. et al. (2014). Generation of gene-modified cynomolgus monkey via Cas9/RNA-mediated gene targeting in one-cell embryos. Cell 156, 836-843. http://dx.doi. org/10.1016/j.cell.2014.01.027

Olson, H., Betton, G., Robinson, D. et al. (2000). Concordance of the toxicity of pharmaceuticals in humans and in animals. Regul Toxicol Pharmacol 32, 56-67. http://dx.doi. org/10.1006/rtph.2000.1399

Smith, A. M. and Dragunow, M. (2014). The human side of microglia. Trends Neurosci 37, 125-135. http://dx.doi. org/10.1016/j.tins.2013.12.001

van der Putten, C., Zuiderwijk-Sick, E. A., van Straalen, L. et al. (2009). Differential expression of adenosine A3 receptors controls adenosine A2A receptor-mediated inhibition of TLR responses in microglia. J Immunol 182, 7603-7612. http:// dx.doi.org/10.4049/jimmunol.0803383

van der Putten, C., Kuipers, H. F., Zuiderwijk-Sick, E. A. et al. (2012). Statins amplify TLR-induced responses in microglia via inhibition of cholesterol biosynthesis. Glia 60, 43-52. http://dx.doi.org/10.1002/glia.21245

van Meer, P. J., Kooijman, M., van der Laan, J. W. et al. (2013). The value of non-human primates in the development of monoclonal antibodies. Nat Biotechnol 31, 882-883. http://dx.doi. org/10.1038/nbt.2709

Vernes, M. K. and Louwerse, A. L. (2010). BPRC's Enrichment Manual for Macaques and Marmosets. http://bit.ly/1mu9Zp3

Zuiderwijk-Sick, E. A., van der Putten, C., Bsibsi, M. et al. (2007). Differentiation of primary adult microglia alters their response to TLR8-mediated activation but not their capacity as APC. Glia 55, 1589-1600. http://dx.doi.org/10.1002/ glia.20572

\section{Acknowledgements}

Part of this work was supported by EUPRIM-Net under the EU grant agreement 262443 of the $7^{\text {th }}$ Framework Programme.

\section{Correspondence to}

Jeffrey J. Bajramovic, PhD

Lange Kleiweg 161

2288GJ Rijswijk

The Netherlands

Phone: +31 152842722

e-mail: bajramovic@bprc.nl 